\title{
Second-order solution of Saint-Venant's problem for an elastic bar predeformed in flexure ${ }^{\text {th }}$
}

\author{
R.C. Batra ${ }^{\mathrm{a}, *}$, F. dell'Isola ${ }^{\mathrm{b}}$, G.C. Ruta ${ }^{\mathrm{b}}$ \\ ${ }^{a}$ Department of Engineering Science and Mechanics, Virginia Polytechnic Institute and State University, Mail Code 0219, \\ Blacksburg, VA 24061, USA \\ bipartimento di Ingegneria Strutturale e Geotecnica, Università di Roma 'La Sapienza', 00184 Roma, Italy
}

Received 30 July 2004; accepted 4 August 2004

\begin{abstract}
We use the method of Signorini's expansion to analyze the Saint-Venant problem for an isotropic and homogeneous second-order elastic prismatic bar predeformed by an infinitesimal amount in flexure. The centroid of one end face of the bar is rigidly clamped. The complete solution of the problem is expressed in terms of ten functions. For a general cross-section, explicit expressions for most of these functions are given; the remaining functions are solutions of well-posed plane elliptic problems. However, for a bar of circular cross-section, all of these functions are evaluated and a closed form solution of the 2nd-order problem is given. The solution contains six constants which characterize the second-order flexure, bending, torsion and extension of the bar. It is found that when the total axial force vanishes, the second-order axial deformation is not zero; it represents a generalized Poynting effect. The second-order elasticities affect only the second-order axial force. (C)
\end{abstract}

Keywords: Signorini's expansion; Second-order elasticity; Prismatic body; Plane-elliptic problems

\section{Introduction}

Poynting [1] discovered that the elongation of a prismatic elastic body deformed by applying equal and opposite torques at the end faces is proportional

\footnotetext{
R.C. Batra dedicates this work to Professor Niall Horgan on his 60th Birthday.

* Corresponding author. Tel.: +5402316051; fax: 5402314574 .

E-mail addresses: rbatra@vt.edu (R.C. Batra), francesco.dellisola@uniroma1.it (F. dell'Isola), giuseppe.ruta@uniroma1.it (G.C. Ruta).
}

to the square of the angle of twist of the bar. Since then there have been many works to qualitatively and quantitatively explain this phenomenon. For example, Rivlin [2] used the second-order theory of elasticity and found that the elongation of a circular bar deformed by applying twisting moments at the end faces depended only upon the second-order elasticities. Truesdell and Noll [3] and Wang and Truesdell [4] have summarized these and other related works.

The problem of bending, torsion, extension and flexure of a prismatic bar is usually known as the Saint- 
Venant problem [5,6]. Iesan [7-10] has analyzed the Saint-Venant problem for anisotropic and microstretch linear elastic solids. Dell'Isola and Rosa [11] and Davi [12] have studied the problem for piezoelectric bars. Recently, dell'Isola et al. $[13,14]$ used the secondorder theory of elasticity to analyze the Saint-Venant problem for a bar predeformed by an infinitesimal amount in either torsion or bending. They used the method of Signorini's expansion [15] to find a secondorder solution and employed Green and Adkins' [16] result that the loads for higher-order problems automatically satisfy the compatibility conditions if the centroid of one end-face of the rod is rigidly clamped. Batra et al. [17] and Vidoli et al. [18] have extended these results to a transversely isotropic piezoelectric bar with the centroidal axis of the bar as the axis of transverse isotropy. We note that Capriz and Podio Guidguli [19] have characterized implications of the Fredholm-type conditions for the existence of Signorini's expansion. They found that for an initially stress free body these generally non-linear algebraic conditions reduce to a sequence of linear systems. They give an example involving infinitesimal deformations superimposed upon a finitely stressed body for which the Signorini expansion method fails.

Here we study the Saint-Venant problem for a homogeneous and isotropic second-order elastic prismatic bar predeformed by an infinitesimal amount in flexure. This problem is very challenging because the solution for the infinitesimal flexure involves twisting and warping of a cross-section of the bar as well as bending of the bar. It is found that for a prismatic bar of general cross-section the second-order Poisson effect, not of the Saint-Venant type, is proportional to $z^{4}$ where $z$ is the distance of a point from the end face whose centroid is assumed to be clamped. The second-order effects also depend upon the first-order elasticities.

\section{Formulation of the problem}

We study quasistatic deformations of a homogeneous and isotropic prismatic body of uniform crosssection $\mathscr{A}$ and length $\ell$ occupying the domain $\mathscr{A} \times$ $[0, \ell]$ in the unstressed reference configuration. We assume that the body is made of a second-order elastic material. In the absence of body forces, its deforma- tions are governed by the balance of linear momentum and the balance of moment of momentum. That is,

$$
\begin{aligned}
\operatorname{Div} \mathbf{T} & =\mathbf{0}, \\
\mathbf{T F}^{\mathrm{T}} & =\mathbf{F T}^{\mathrm{T}},
\end{aligned}
$$

where

$$
\begin{aligned}
\mathbf{T}= & \mu\left[\left(\alpha_{1} I_{\mathbf{E}}+2 \mathbf{E}+\frac{\alpha_{1}}{2}\left(I_{\mathbf{H}} \mathbf{H}^{\mathrm{T}}+3 I_{\mathbf{E}}^{2}\right)+\alpha_{3} I_{\mathbf{E}}^{2}\right.\right. \\
& \left.+\alpha_{4} I_{\mathbf{E}}\right)+\left(\alpha_{5}+2\right) I_{\mathbf{E}} \mathbf{E}-\alpha_{1} I_{\mathbf{E}} \mathbf{H}^{\mathrm{T}} \\
& \left.-\left(\mathbf{H}^{\mathrm{T}}\right)^{2}+\alpha_{6} \mathbf{E}^{2}\right]
\end{aligned}
$$

$\mathbf{H}=\operatorname{Grad} \mathbf{u}, \mathbf{u}=\mathbf{x}-\mathbf{X}, \mathbf{E}=\left(\mathbf{H}+\mathbf{H}^{\mathrm{T}}\right) / 2$,

$I_{\mathbf{E}}=\operatorname{tr} \mathbf{E}, I I_{\mathbf{E}}=\frac{1}{2}\left(I_{\mathbf{E}}^{2}-I_{\mathbf{E}^{2}}\right)$.

Here $\mathbf{T}$ is the first Piola-Kirchhoff stress tensor, $\mathbf{F}$ the deformation gradient, $\mathbf{u}$ the displacement of a material point, $\mathbf{x}$ the present position of the material point that occupied place $\mathbf{X}$ in the reference configuration, Div and Grad denote the three-dimensional divergence and gradient operators with respect to coordinates in the reference configuration, $\mu$ is the shear modulus, $\alpha_{1}=$ $\lambda / \mu, \lambda$ is the other Lamé constant, and $\alpha_{3}, \alpha_{4}, \alpha_{5}$ and $\alpha_{6}$ are non-dimensional second-order elasticities of the material. The reader is referred to Wang and Truesdell [4] and Truesdell and Noll [3] for details of deriving the constitutive relation (3) and for references to other authors who have derived it. Following $[3,4]$ we use the direct notation. Within terms of $\mathrm{O}\left(\mathbf{H}^{2}\right)$, the constitutive relation (3) identically satisfies Eq. (2). Substitution from (3) into (1) gives a system of coupled non-linear partial differential equations to be solved for the three components of the displacement $\mathbf{u}$. Having found $\mathbf{u}$, the balance of mass can be used to ascertain the mass density in the present configuration.

We assume that the prismatic body is loaded by applying tractions only to its end faces. Thus

$$
\begin{aligned}
& \mathbf{T N}=\mathbf{f} \text { on } \mathscr{A}_{0} \text { and on } \mathscr{A}_{\ell}-N_{C}, \\
& \mathbf{T N}=\mathbf{0} \text { on } \partial \mathscr{A} \times[0, \ell], \\
& \mathbf{u}=\mathbf{0}, \mathbf{H}-\mathbf{H}^{\mathrm{T}}=\mathbf{0} \text { at } C .
\end{aligned}
$$

Here $\mathscr{A}_{0}=A \times\{0\}$ and $\mathscr{A}_{\ell}=A \times\{\ell\}$ are the end faces of the prismatic body, $\mathbf{N}$ is an outward unit normal at a point on the boundary in the reference configuration, $C$ is the centroid of the cross-section $\mathscr{A}_{\ell}, N_{C}$ is a small neighborhood of $C$ in the plane $\mathscr{A}_{\ell}$ and $\partial \mathscr{A}$ is the boundary of $\mathscr{A}$. Green and Adkins [16] have shown 
that under conditions (7), the method of Signorini's expansion [15] produces loads which automatically satisfy compatibility conditions for the boundary-value problems of different orders to be well-posed. In the Saint-Venant semi-inverse method, surface tractions $\mathbf{f}$ are determined from the solution of the problem.

The integrability conditions for the boundary-value problem defined by Eqs. (1)-(7) are

$$
\begin{aligned}
& \left(\int_{\mathscr{A}} \mathbf{f} \mathrm{d} A\right)^{\prime}=\mathbf{0}, \\
& \left(\int_{\mathscr{A}} \mathbf{x} \wedge \mathbf{f} \mathrm{d} A\right)^{\prime}+\left.\mathbf{x}\right|_{\mathbf{r}=\mathbf{0}} ^{\prime} \wedge \int_{\mathscr{A}} \mathbf{f} \mathrm{d} A=\mathbf{0},
\end{aligned}
$$

where a prime denotes differentiation with respect to the axial coordinate $z, \mathbf{a} \wedge \mathbf{b}=\mathbf{b} \otimes \mathbf{a}-\mathbf{a} \otimes \mathbf{b}, \mathbf{a} \vee$ $\mathbf{b}=\mathbf{a} \otimes \mathbf{b}+\mathbf{b} \otimes \mathbf{a},(\mathbf{a} \otimes \mathbf{b}) \mathbf{d}=(\mathbf{b} \cdot \mathbf{d}) \mathbf{a}$ for arbitrary vectors $\mathbf{a}, \mathbf{b}$ and $\mathbf{d}$. With the origin at the centroid of the cross-section $\mathscr{A}_{0}$, we set

$\mathbf{X}=\mathbf{r}+z \mathbf{e}, \quad \mathbf{u}=w \mathbf{e}+\mathbf{v}$,

where $\mathbf{e}$ is a unit vector along the centroidal axis of the prismatic body, $\mathbf{r}$ the in-plane position vector of a point, and $w$ and $\mathbf{v}$ equal the axial and the in-plane components, respectively, of the displacement $\mathbf{u}$ of a point.

\section{Signorini's expansion}

In Signorini's method, the displacement $\mathbf{u}$ and the surface traction $\mathbf{f}$ are assumed to have a series expansion

$\mathbf{u}=\eta \dot{\mathbf{u}}+\eta^{2} \ddot{\mathbf{u}}+\cdots, \quad \mathbf{f}=\eta \dot{\mathbf{f}}+\eta^{2} \ddot{\mathbf{f}}+\cdots$,

where $\eta$, a small parameter in the expansion, is associated with the curvature of the bar predeformed in flexure. Note that $\dot{\mathbf{u}}$ is a solution of the linear problem corresponding to Eqs. (1)-(7) and $\ddot{\mathbf{u}}$ is a correction because of the consideration of second-order effects. Thus a superimposed dot indicates the order of the solution rather than the time derivative. Substitution from (10) $)_{1}$ into (4) and the result into (3) yields

$\mathbf{T}=\eta \dot{\mathbf{T}}+\eta^{2} \ddot{\mathbf{T}}+\cdots$, where

$$
\begin{aligned}
\dot{\mathbf{T}}= & 2 \mu \dot{\mathbf{E}}+\lambda(\operatorname{tr} \dot{\mathbf{E}}) \mathbf{1}, \ddot{\mathbf{T}}=\ddot{\overline{\mathbf{T}}}+\ddot{\mathbf{T}}_{s}, \\
\ddot{\overline{\mathbf{T}}}= & 2 \mu \ddot{\mathbf{E}}+\lambda(\operatorname{tr} \ddot{\mathbf{E}}) \mathbf{1}, \\
\ddot{\mathbf{T}}_{s}= & \mu\left[\frac{\alpha_{1}}{2} I_{\dot{\mathbf{H}}} \dot{\mathbf{H}}^{\mathrm{T}}+\left(2+\alpha_{3}\right) I_{\dot{\mathbf{E}}}^{2}+\alpha_{4} I I_{\dot{\mathbf{E}}} \mathbf{1}\right. \\
& +\left(\alpha_{5}+2\right) I_{\dot{\mathbf{E}}} \dot{\mathbf{E}}-\alpha_{1} I_{\dot{\mathbf{E}}} \dot{\mathbf{H}}^{\mathrm{T}} \\
& \left.-\left(\dot{\mathbf{H}}^{\mathrm{T}}\right)^{2}+\alpha_{6} \dot{\mathbf{E}}^{2}\right] .
\end{aligned}
$$

Note that $\dot{\mathbf{T}}$ and $\ddot{\overline{\mathbf{T}}}$ are symmetric tensors, expressions $(12)_{1}$ and $(12)_{3}$ for them are alike, and $\ddot{\mathbf{T}}$ is not symmetric. Eq. (12) $)_{1}$ is Hooke's law for a linear elastic isotropic body. From (1), (5), (6), (7), (10) 2 and (11) we conclude that

$\operatorname{Div} \ddot{\mathbf{T}}=\mathbf{0}$ in $\mathscr{A} \times[0, \ell]$,

$\ddot{\mathbf{T}} \mathbf{N}=\mathbf{0}$ on $\partial \mathscr{A} \times[0, \ell]$,

$\ddot{\mathbf{T}} \mathbf{N}=\ddot{\mathbf{f}} \quad$ on $\mathscr{A}_{0}$ and $\mathscr{A}_{\ell}-N_{C}$,

$\ddot{w}=0, \ddot{\mathbf{v}}=\mathbf{0}, \quad \ddot{\mathbf{H}}-\ddot{\mathbf{H}}^{\mathrm{T}}=\mathbf{0}$ on $N_{C}$.

In a way analogous to $(9)_{2}$ we write

$$
\begin{aligned}
& \dot{\mathbf{u}}=\dot{w} \mathbf{e}+\dot{\mathbf{v}}, \\
& \dot{\mathbf{E}}=\dot{\varepsilon} \mathbf{e} \otimes \mathbf{e}+\dot{\mathbf{g}} \otimes \mathbf{e}+\mathbf{e} \otimes \dot{\mathbf{g}}+\dot{\hat{\mathbf{E}}}, \\
& \dot{\mathbf{T}}=\dot{\sigma} \mathbf{e} \otimes \mathbf{e}+\dot{\mathbf{t}} \otimes \mathbf{e}+\mathbf{e} \otimes \dot{\mathbf{t}}+\dot{\hat{\mathbf{T}}}, \\
& \ddot{\overline{\mathbf{T}}}=\ddot{\bar{\sigma}} \mathbf{e} \otimes \mathbf{e}+\ddot{\ddot{\mathbf{t}}} \otimes \mathbf{e}+\mathbf{e} \otimes \ddot{\overrightarrow{\mathbf{t}}}+\ddot{\overline{\mathbf{T}}},
\end{aligned}
$$

where

$\dot{\hat{\mathbf{E}}}=(\operatorname{grad} \dot{\mathbf{v}})_{s} \equiv\left(\operatorname{grad} \dot{\mathbf{v}}+(\operatorname{grad} \dot{\mathbf{v}})^{\mathrm{T}}\right) / 2$,
$\dot{\varepsilon}=\dot{w}^{\prime} \equiv \partial \dot{w} / \partial z, \quad \dot{\mathbf{g}}=\left(\dot{\mathbf{v}}^{\prime}+\operatorname{grad} \dot{w}\right)$,

and grad (div) is the two-dimensional gradient (divergence) operator with respect to coordinates in the cross-section $\mathscr{A}$. In (14) $\varepsilon$ is the axial strain, $\sigma$ the axial stress, $\mathbf{t}$ the shear stress vector on a plane perpendicular to the axis of the bar, $\mathbf{g}$ the corresponding shear strain vector, $\hat{\mathbf{E}}$ the two-dimensional in-plane symmetric strain tensor appropriate for infinitesimal deformations, and $\hat{\mathbf{T}}$ the corresponding in-plane twodimensional stress tensor. We decompose $\ddot{\mathbf{u}}$ and $\ddot{\mathbf{E}}$ in a way similar to that for $\dot{\mathbf{u}}$ and $\dot{\mathbf{E}}$ given in (14) and $(14)_{2}$. 
We assume that initial deformations of the bar correspond to the Saint-Venant flexure $[20,21]$. Thus

$$
\begin{aligned}
\dot{\mathbf{u}}= & \left\{z \dot{\mathbf{v}}_{0}^{\prime}+\frac{z^{3}}{6} \dot{\mathbf{c}}^{\prime}+z \dot{\tau}^{F}(* \mathbf{r})+z \mathbf{K} \dot{\mathbf{c}}^{\prime}\right. \\
& +\left[\dot{w}_{0}-\left(\frac{z^{2}}{2} \dot{\mathbf{c}}^{\prime}+\dot{\mathbf{v}}_{0}^{\prime}\right) \cdot \mathbf{r}\right. \\
& \left.\left.+\dot{\tau}^{F} \phi+\dot{\mathbf{c}}^{\prime} \cdot \mathbf{h}\right] \mathbf{e}\right\},
\end{aligned}
$$

where

$\mathbf{K}=\frac{\nu}{2}(\mathbf{r} \otimes \mathbf{r}-(* \mathbf{r}) \otimes(* \mathbf{r}))$,

$\dot{w}_{0}=\dot{\tau}^{F} \phi(\mathbf{r})+\dot{\chi}(\mathbf{r}), \quad \dot{\chi}=\dot{\mathbf{c}}^{\prime} \cdot \mathbf{h}$,

$\dot{\mathbf{v}}_{0}^{\prime}=\operatorname{grad}\left(\dot{\tau}^{F} \phi+\dot{\chi}\right)$,

$\dot{\tau}^{F}=v\left(* \dot{\mathbf{c}}^{\prime}\right) \cdot \int_{\mathscr{P}} \hat{\psi} \mathbf{r} \mathrm{d} A / \int_{\mathscr{P}} \hat{\psi} \mathrm{d} A$,

$v=\lambda / 2(\lambda+\mu)$ is Poisson's ratio, $\hat{\psi}$ the continuous continuation of the Prandtl function from $\mathscr{A}$ to the entire plane $\mathscr{P}$ containing $\mathscr{A}, \mathbf{c}^{\prime}$ the curvature of the deformed bar, $\mathbf{K}$ is the anticlastic curvature tensor, $* \mathbf{r}$ is defined by $* \mathbf{r}=\mathbf{e} \times \mathbf{r}, \phi$ is the Saint-Venant warping function, and $\mathbf{h}$ the solution of

$\Delta_{R} \mathbf{h}=2 \mathbf{r}$ in $\mathscr{A},(\operatorname{grad} \mathbf{h}) \mathbf{N}=-\mathbf{K N}$ on $\partial \mathscr{A}$.

Here $\Delta_{R}$ is the two-dimensional Laplace operator and $\mathbf{N}$ is the outward unit normal to $\partial \mathscr{A}$. In (16) with $\dot{\tau}^{F}$ given by $(17)_{5}$ we have used Trefftz's [20] solution for flexure. With definitions

$\delta=\dot{\mathbf{c}}^{\prime} \cdot \mathbf{r}, \quad \theta=v\left(* \dot{\mathbf{c}}^{\prime}\right) \cdot \mathbf{r}+\dot{\tau}^{F}$,

$\dot{\mathbf{g}}=\left[\dot{\tau}^{F}(* \mathbf{r}+\operatorname{grad} \phi)+(\operatorname{grad} \mathbf{h}+\mathbf{K})^{\mathrm{T}} \dot{\mathbf{c}}^{\prime}\right]$,

we obtain the following relations from the displacement field (16):

$$
\begin{aligned}
\dot{\mathbf{H}}= & -z \delta \mathbf{e} \otimes \mathbf{e}+\mathbf{e} \wedge\left(\dot{\mathbf{v}}_{0}^{\prime}+\frac{z^{2}}{2} \dot{\mathbf{c}}^{\prime}\right) \\
& +\left(\dot{\tau}^{F}(* \mathbf{r})+\mathbf{K} \dot{\mathbf{c}}^{\prime}\right) \otimes \mathbf{e} \\
& +\mathbf{e} \otimes\left(\dot{\tau}^{F} \operatorname{grad} \phi+(\operatorname{grad} \mathbf{h})^{\mathrm{T}} \dot{\mathbf{c}}^{\prime}\right) \\
& +v \delta \hat{\mathbf{I}}+[z \theta *],
\end{aligned}
$$

$$
\dot{\mathbf{E}}=-z \delta \mathbf{e} \otimes \mathbf{e}+\frac{1}{2} \mathbf{e} \vee \dot{\mathbf{g}}+z v \delta \hat{\mathbf{I}},
$$

$$
\begin{aligned}
\dot{\mathbf{H}} \dot{\mathbf{H}}^{\mathrm{T}}= & \left(z^{2} \delta^{2}+\|\operatorname{grad} \dot{w}\|^{2}\right) \mathbf{e} \otimes \mathbf{e} \\
& +z \delta\left(v \operatorname{grad} \dot{w}-\dot{\mathbf{v}}^{\prime}\right) \vee \mathbf{e} \\
& +z \theta(* \operatorname{grad} \dot{w}) \vee \mathbf{e}+\dot{\mathbf{v}}^{\prime} \otimes \dot{\mathbf{v}}^{\prime} \\
& +z^{2}\left(v^{2} \delta^{2}+\theta^{2}\right) \hat{\mathbf{I}}, \\
\dot{\mathbf{E}}^{2}= & \left(z^{2} \delta^{2}+\frac{1}{4}\|\dot{\mathbf{g}}\|^{2}\right) \mathbf{e} \otimes \mathbf{e}+\frac{z \delta}{2}(v-1) \dot{\mathbf{g}} \vee \mathbf{e} \\
& +\frac{1}{4} \dot{\mathbf{g}} \otimes \dot{\mathbf{g}}+z^{2} v^{2} \delta^{2} \hat{\mathbf{I}},
\end{aligned}
$$

$\left(\dot{\mathbf{H}}^{\mathrm{T}}\right)^{2}=\left(z^{2} \delta^{2}+\dot{\mathbf{v}} \cdot \operatorname{grad} \dot{w}\right) \mathbf{e} \otimes \mathbf{e}+z \delta(v-1)\left(\mathbf{e} \otimes \dot{\mathbf{v}}^{\prime}\right.$

$$
\begin{aligned}
& +\operatorname{grad} \dot{w} \otimes \mathbf{e})+\operatorname{grad} \dot{w} \otimes \dot{\mathbf{v}}^{\prime} \\
& +z \theta\left(\mathbf{e} \otimes * \dot{\mathbf{v}}^{\prime}-* \operatorname{grad} \dot{w} \otimes \mathbf{e}\right) \\
& +z^{2}\left(v^{2} \delta^{2}-\theta^{2}\right) \hat{\mathbf{I}}-2 v z^{2} \theta \delta * .
\end{aligned}
$$

Here $\hat{\mathbf{I}}$ is the two-dimensional identity tensor, and $\|\mathbf{a}\|$ equals the length of vector a. The two-dimensional identity matrix $\hat{\mathbf{I}}$ is extended to a three-dimensional matrix by adding zeroes in the third row and the third column, and the rotation operator $*$ is also similarly extended. In a rectangular Cartesian coordinate system with e pointing along the 3 -axis, elements of the first, the second and the third rows of the $3 \times 3$ matrix corresponding to the $*$ operator are $0,-1,0 ; 1,0,0 ; 0,0,0$ respectively. Substitution from (20) into (12) and some simplification gives

$$
\begin{aligned}
\ddot{\mathbf{T}}_{s}= & {\left[\lambda z^{2}\left(\gamma_{1} \delta^{2}+\theta^{2}\right)-(\lambda+\mu) \dot{\mathbf{v}}^{\prime} \cdot \operatorname{grad} \dot{w}+\left(\gamma_{2}\|\dot{\mathbf{g}}\|^{2}\right.\right.} \\
& \left.\left.+\mu \beta_{1} z^{2} \delta^{2}\right)\right] \mathbf{e} \otimes \mathbf{e}+\frac{1}{4} \mu \alpha_{6} \dot{\mathbf{g}} \otimes \dot{\mathbf{g}}+\frac{1}{2} \mu \beta_{2} z \delta \dot{\mathbf{g}} \vee \mathbf{e} \\
& -\mu \operatorname{grad} \dot{w} \otimes \dot{\mathbf{v}}^{\prime}+\frac{1}{2} z \delta E\left(\mathbf{e} \otimes \dot{\mathbf{v}}^{\prime}+\operatorname{grad} \dot{w} \otimes \mathbf{e}\right) \\
& -z \theta \mu\left(\mathbf{e} \otimes * \dot{\mathbf{v}}^{\prime}-* \operatorname{grad} \dot{w} \otimes \mathbf{e}\right)+\Omega \hat{\mathbf{I}},
\end{aligned}
$$

where $E=\mu(3 \lambda+2 \mu) /(\lambda+\mu)$ is Young's modulus for the material of the body, and

$$
\begin{aligned}
\Omega= & {\left[\lambda\left(\left(\frac{3-6 v+6 v^{2}}{2}\right) z^{2} \delta^{2}-\dot{\mathbf{v}}^{\prime} \cdot \operatorname{grad} \dot{w}\right)\right.} \\
& \left.+(\lambda+\mu) z^{2} \theta^{2}+\frac{1}{4}\left(2 \lambda-\mu \alpha_{4}\right)\|\dot{\mathbf{g}}\|^{2}+\mu \beta_{3} z^{2} \delta^{2}\right],
\end{aligned}
$$




$$
\begin{aligned}
\gamma_{1}= & \left(1-4 v+10 v^{2}\right) / 2, \\
\gamma_{2}= & \left(2 \lambda-\mu\left(\alpha_{6}+\alpha_{4}\right)\right) / 4 \\
\beta_{1}= & 1-4 v+\alpha_{3}(2 v-1)^{2}+\alpha_{4} v(v-2) \\
& +\alpha_{5}(1-2 v)+\alpha_{6}, \\
2 \beta_{2}= & \left(\alpha_{5}+2\right)(-2 v+1)+\alpha_{6}(-v+1), \\
\beta_{3}= & \beta_{1}-\left(\alpha_{5}+2\right)\left(1-v-2 v^{2}\right) \\
& +\left(\alpha_{6}-1\right)\left(1+v^{2}\right)
\end{aligned}
$$

Eqs. (13) can now be written as

$$
\begin{aligned}
& \ddot{\sigma}^{\prime}+\operatorname{div} \ddot{\mathbf{t}}=\eta^{2}\left\{(\lambda+\mu)\left(\dot{\mathbf{v}}^{\prime} \cdot \operatorname{grad} \dot{w}\right)^{\prime}\right. \\
& -2\left(\lambda \gamma_{1}+\mu \beta_{1}\right) z \delta^{2} \\
& -2 \lambda z \theta^{2}+\mu z \operatorname{div}\left(* \dot{\mathbf{v}}^{\prime} \theta\right) \\
& -\frac{E}{2} z \operatorname{div}\left(\dot{\mathbf{v}}^{\prime} \delta\right) \\
& \left.-\frac{\mu \beta_{2}}{2} z \operatorname{div}(\dot{\mathbf{g}} \delta)\right\} \text { in } \mathscr{A} \times[0, \ell] \\
& \ddot{\mathbf{t}}^{\prime}+\operatorname{div}(\ddot{\overline{\hat{\mathbf{T}}}})=\eta^{2}\left\{-\frac{E}{2} \delta(z \operatorname{grad} \dot{w})^{\prime}-\frac{\delta \mu \beta_{2}}{2} \dot{\mathbf{g}}\right. \\
& +\mu \operatorname{div}\left(\operatorname{grad} \dot{w} \otimes \dot{\mathbf{v}}^{\prime}\right) \\
& -\frac{\mu \alpha_{6}}{4} \operatorname{div}(\dot{\mathbf{g}} \otimes \dot{\mathbf{g}})+\operatorname{grad} \Omega \\
& \left.-\mu \theta(z * \operatorname{grad} \dot{w})^{\prime}\right\} \text { in } \mathscr{A} \times[0, \ell],
\end{aligned}
$$

The integrability conditions (8) imply that

$$
\begin{aligned}
& \int_{\mathscr{A}} \ddot{\sigma} \mathrm{d} A+\eta^{2}\left\{z^{2}\left(\lambda \gamma_{1}+\mu \beta_{1}\right)\left(\mathbf{J} * \dot{\mathbf{c}}^{\prime}\right) \cdot\left(* \dot{\mathbf{c}}^{\prime}\right)\right. \\
& \quad+\lambda z^{2}\left(\dot{\tau}^{F} A+v^{2}\left(\mathbf{J} \dot{\mathbf{c}}^{\prime}\right) \cdot \dot{\mathbf{c}}^{\prime}\right) \\
& \quad-(\lambda+\mu) \int_{\mathscr{A}}\left(\dot{\mathbf{v}}^{\prime} \cdot \operatorname{grad} \dot{w}\right) \mathrm{d} A+\gamma_{2}\left(\dot{\tau}^{F}\right)^{2}\left(J_{0}-D\right) \\
& \left.\left.\quad+2 \dot{\tau}^{F} \dot{\mathbf{c}}^{\prime} \cdot \mathbf{p}+\dot{\mathbf{c}}^{\prime} \cdot \mathbf{Z} \dot{\mathbf{c}}^{\prime}\right)\right\}=\text { const. },
\end{aligned}
$$

$$
\begin{aligned}
& \int_{\mathscr{A}} \ddot{\mathbf{t}} \mathrm{d} A+\eta^{2}\left\{z \left[\frac{E}{2} \int_{\mathscr{A}}(\operatorname{grad} \dot{w} \otimes \mathbf{r}) \dot{\mathbf{c}}^{\prime} \mathrm{d} A\right.\right. \\
& \quad+\mu \int_{\mathscr{A}} \theta(* \operatorname{grad} \dot{w}) \mathrm{d} A \\
& \left.\left.\quad+\frac{\mu \beta_{2}}{2} \int_{\mathscr{A}}(\dot{\mathbf{g}} \otimes \mathbf{r}) \dot{\mathbf{c}}^{\prime} \mathrm{d} A\right]\right\}=\text { const. } \\
& \int_{\mathscr{A}}[(\mathbf{r}+z \mathbf{e}) \wedge \ddot{\mathbf{T}} \mathbf{e}+(\dot{\mathbf{u}} \wedge \dot{\mathbf{T}} \mathbf{e})] \mathrm{d} A=\text { const. }
\end{aligned}
$$

where

$$
\begin{aligned}
& \mathbf{J}=\int_{\mathscr{A}} \mathbf{r} \otimes \mathbf{r} \mathrm{d} A, \quad J_{0}=\operatorname{tr} \mathbf{J}, \\
& D=\int_{\mathscr{A}}(* \operatorname{grad} \phi) \cdot \mathbf{r} \mathrm{d} A \\
& \mathbf{p}=\int_{\mathscr{A}} \mathbf{K} \operatorname{grad} \phi \mathrm{d} A-\frac{v}{2} \int_{\mathscr{A}}\|\mathbf{r}\|^{2}(* \mathbf{r}) \mathrm{d} A, \\
& \mathbf{Z}=\int_{\mathscr{A}}(\operatorname{grad} \mathbf{h}+\mathbf{K})(\operatorname{grad} \mathbf{h}+\mathbf{K})^{\mathrm{T}} \mathrm{d} A .
\end{aligned}
$$

Note that even though $z$ appears explicitly in some of the terms on the left-hand sides of (24), values of expressions are independent of $z$. In (24) and (25) $\mathscr{A}$ is the area of cross-section of the bar, $\mathbf{J}$ the inertia tensor, $J_{0}$ the polar moment of inertia, and $\mu\left(J_{0}-D\right)$ the torsional rigidity of the first-order Saint-Venant torsional problem.

Eqs. (23)-(25) define the second-order Saint-Venant problem for an elastic prismatic bar predeformed by an infinitesimal amount in flexure.

\section{Saint-Venant solutions}

Assuming that $\ddot{\mathbf{u}}(\mathbf{r}, z)$ is a polynomial in $z$, we write it as

$$
\begin{aligned}
\ddot{\mathbf{u}}(\mathbf{r}, z)= & \mathbf{u}_{0}(\mathbf{r})+z \mathbf{u}_{1}(\mathbf{r})+\frac{z^{2}}{2 !} \mathbf{u}_{2}(\mathbf{r}) \\
& +\frac{z^{3}}{3 !} \mathbf{u}_{3}(\mathbf{r})+\cdots,
\end{aligned}
$$

where, to simplify the notation, we have dropped the superimposed double dots on $\mathbf{u}_{0}, \mathbf{u}_{1}, \mathbf{u}_{2}$ etc. appearing on the right-hand side of (26). There is no implication that $z$ is small. The solution (26) will not capture well the boundary layer effects, if any, near the end faces $z=0$ and $z=\ell$. However, the solution should be quite good away from the end faces. The preceding 
statements tacitly presume that Saint-Venant's principle holds for a second-order elastic prismatic bar; however, to the best of our knowledge, this has not been proved. Noting that the right-hand sides of $(23)_{1}-(23)_{4}$ are polynomials of degree at most four in $z$, we obtain for $\mathbf{u}_{6}, \mathbf{u}_{7} \ldots$ etc. equations analogous to those in linear elasticity with zero body force and zero traction boundary conditions. Because of the clamping conditions (7) or $(13)_{4}-(13)_{6}$ at the centroid of one end face, and the uniqueness of solutions in linear elasticity, $\mathbf{u}_{6}=\mathbf{u}_{7}=\cdots=\mathbf{0}$, and for $\mathbf{u}_{5}$ we obtain

$\mathbf{u}_{5}=w_{5}^{0} \mathbf{e}+\mathbf{v}_{5}^{0}+\omega_{5}^{0}(* \mathbf{r})$.

Throughout this paper, quantities with superscript zero denote constants; $w_{5}^{0}$ represents the axial elongation of points of the bar, $\mathbf{v}_{5}^{0}$ the in-plane displacements, and $\omega_{5}^{0}$ the angular twist. Note that each of these quantities is to be multiplied with $z^{5} / 5$ !. Eqs. (26), (9) 2 and (27) yield

$$
\begin{aligned}
\ddot{w}(\mathbf{r}, z)= & \frac{z^{5}}{5 !} w_{5}^{0}+\frac{z^{4}}{4 !} w_{4}(\mathbf{r})+\frac{z^{3}}{3 !} w_{3}(\mathbf{r}) \\
& +\frac{z^{2}}{2 !} w_{2}(\mathbf{r})+z w_{1}(\mathbf{r})+w_{0}(\mathbf{r}), \\
\ddot{\mathbf{v}}(\mathbf{r}, z)= & \frac{z^{5}}{5 !}\left(\mathbf{v}_{5}^{0}+\omega_{5}^{0}(* \mathbf{r})\right)+\frac{z^{4}}{4 !} \mathbf{v}_{4}(\mathbf{r}) \\
& +\frac{z^{3}}{3 !} \mathbf{v}_{3}(\mathbf{r})+\frac{z^{2}}{2 !} \mathbf{v}_{2}(\mathbf{r})+z \mathbf{v}_{1}(\mathbf{r})+\mathbf{v}_{0}(\mathbf{r}) .
\end{aligned}
$$

Equations for the determination of $w_{4}$ and $\mathbf{v}_{4}$ are

$\Delta_{R} w_{4}=0$ in $\mathscr{A}$,

$\mu \Delta_{R} \mathbf{v}_{4}+(\lambda+\mu) \operatorname{grad} \operatorname{div} \mathbf{v}_{4}=\mathbf{0}$ in $\mathscr{A}$,

$\left(\operatorname{grad} w_{4}\right) \cdot \mathbf{N}=-\left(\mathbf{v}_{5}^{0}+\omega_{5}^{0}(* \mathbf{r})\right) \cdot \mathbf{N}$ on $\mathscr{A}$,

$\left[2 \mu\left(\operatorname{grad} \mathbf{v}_{4}\right)_{s}+\lambda\left(\operatorname{div} \mathbf{v}_{4}\right) \hat{\mathbf{I}}\right] \mathbf{N}$

$$
=-6 \eta^{2}\left[\mu \dot{\mathbf{c}}^{\prime} \otimes \dot{\mathbf{c}}^{\prime}+\lambda\left\|\dot{\mathbf{c}}^{\prime}\right\|^{2} \hat{\mathbf{I}}\right] \mathbf{N} \text { on } \partial \mathscr{A},
$$

and have the solution

$$
\begin{aligned}
w_{4}(\mathbf{r})= & w_{4}^{0}-\mathbf{v}_{5}^{0} \cdot \mathbf{r}+\omega_{5}^{0} \phi(\mathbf{r}), \\
\mathbf{v}_{4}(\mathbf{r})= & \mathbf{v}_{4}^{0}+\omega_{4}^{0}(* \mathbf{r})-3 \eta^{2}\left[\left(\dot{\mathbf{c}}^{\prime} \otimes \dot{\mathbf{c}}^{\prime}\right) \mathbf{r}\right. \\
& \left.+v\left\|\dot{\mathbf{c}}^{\prime}\right\|^{2} \mathbf{r}\right] .
\end{aligned}
$$

The integrability conditions (24) require that

$w_{5}^{0}=-3 \eta^{2} E\left\|\dot{\mathbf{c}}^{\prime}\right\|^{2} /(\lambda+2 \mu), \omega_{5}^{0}=0$.
Thus

$$
\begin{aligned}
w_{5} & =-\frac{3 E}{(\lambda+2 \mu)} \eta^{2}\left\|\dot{\mathbf{c}}^{\prime}\right\|^{2}, \mathbf{v}_{5}=\mathbf{v}_{5}^{0}, \\
w_{4} & =w_{4}^{0}-\mathbf{v}_{5}^{0} \cdot \mathbf{r}, \\
\mathbf{v}_{4}(\mathbf{r}) & =\mathbf{v}_{4}^{0}+\omega_{4}^{0}(* \mathbf{r})-3 \eta^{2}\left(\dot{\mathbf{c}}^{\prime} \otimes \dot{\mathbf{c}}^{\prime}+v\|\dot{\mathbf{c}}\|^{2} \hat{\mathbf{I}}\right) \mathbf{r} .
\end{aligned}
$$

Governing equations for $w_{3}$ and $\mathbf{v}_{3}$ are

$$
\begin{aligned}
& \Delta_{R} w_{3}=6 v \eta^{2}\left\|\dot{\mathbf{c}}^{\prime}\right\|^{2} \text { in } \mathscr{A} \\
& \mu \Delta_{R} \mathbf{v}_{3}+(\lambda+\mu) \operatorname{grad} \operatorname{div} \mathbf{v}_{3}=\lambda \mathbf{v}_{5}^{0} \text { in } \mathscr{A}, \\
& \left(\operatorname{grad} w_{3}\right) \cdot \mathbf{N}=\left[-\mathbf{v}_{4}^{0}-\omega_{4}^{0}(* \mathbf{r})\right. \\
& +3 \eta^{2}\left(\dot{\tau}^{F} * \dot{\mathbf{c}}^{\prime}\right. \\
& + \\
& \left.+2 v\left(* \dot{\mathbf{c}}^{\prime} \otimes * \dot{\mathbf{c}}^{\prime}\right) \mathbf{r}\right] \cdot \mathbf{N} \text { on } \partial \mathscr{A},
\end{aligned}
$$$$
\begin{aligned}
& {\left[2 \mu\left(\operatorname{grad} \mathbf{v}_{3}\right)_{s}+\lambda\left(\operatorname{div} \mathbf{v}_{3}\right) \hat{\mathbf{I}}\right] \mathbf{N}} \\
& \quad=\lambda\left(\mathbf{v}_{5}^{0} \cdot \mathbf{r}-w_{4}^{0}\right) \mathbf{N} \text { on } \partial \mathscr{A} .
\end{aligned}
$$

The solution of Eqs. (33) is

$$
\begin{aligned}
& w_{3}(\mathbf{r})=w_{3}^{0}-\mathbf{v}_{4}^{0} \cdot \mathbf{r}+\omega_{4}^{0} \phi(\mathbf{r})+3 \eta^{2}\left(* \dot{\mathbf{c}}^{\prime} \cdot \mathbf{r}\right) \theta \\
& \mathbf{v}_{3}(\mathbf{r})=\mathbf{v}_{3}^{0}+\omega_{3}^{0}(* \mathbf{r})-v w_{4}^{0} \mathbf{r}+\mathbf{K} \mathbf{v}_{5}^{0}
\end{aligned}
$$

and the integrability conditions (24) give

$\omega_{4}^{0}=\frac{3 \eta^{2} \dot{\mathbf{q}} \cdot\left(* \dot{\mathbf{c}}^{\prime}\right)}{\mu\left(J_{0}-D\right)}, w_{4}^{0}=0$,

where $\dot{\mathbf{q}}$ is the first-order resultant shear force. Even though the elongation, $w_{4}^{0}$, multiplying $z^{4} / 4$ ! equals zero, the twist multiplying $z^{4} / 4$ ! will not vanish unless $\dot{\mathbf{q}}$ is orthogonal to $* \dot{\mathbf{c}}^{\prime}$, and the total second-order twist can be large for a long bar.

The boundary-value problem for the determination of $\mathbf{v}_{2}$ and $w_{2}$ is

$$
\begin{aligned}
& \Delta_{R} w_{2}=2 \mathbf{v}_{5}^{0} \cdot \mathbf{r} \text { in } \mathscr{A}, \\
& (\lambda+\mu) \operatorname{grad} \operatorname{div} \mathbf{v}_{2}+\mu \Delta_{R} \mathbf{v}_{2} \\
& =\lambda\left(\mathbf{v}_{4}^{0}-\omega_{4}^{0} \operatorname{grad} \phi\right)-\mu \omega_{4}^{0}((* \mathbf{r}) \\
& \quad+\operatorname{grad} \phi)+\eta^{2}\left\{3 \mu v\left\|\dot{\mathbf{c}}^{\prime}\right\|^{2} \mathbf{r}\right. \\
& \quad+(\lambda+\mu) \mathscr{H}\left(\dot{\tau}^{F} \phi+\chi\right) \dot{\mathbf{c}}^{\prime}
\end{aligned}
$$




$$
\begin{aligned}
& +\left(\mu\left(6+v-4 \beta_{3}\right)-4 \lambda \gamma_{3}\right) \delta \dot{\mathbf{c}}^{\prime} \\
& \left.-\frac{3}{2} \lambda\left(* \dot{\mathbf{c}}^{\prime} \otimes * \dot{\mathbf{c}}^{\prime}\right)-4 \lambda \theta * \dot{\mathbf{c}}^{\prime}\right\} \text { in } \mathscr{A},
\end{aligned}
$$

$\left(\operatorname{grad} w_{2}\right) \cdot \mathbf{N}=-\left(\mathbf{v}_{3}^{0}+\omega_{3}^{0}(* \mathbf{r})+\mathbf{K} \mathbf{v}_{5}^{0}\right) \cdot \mathbf{N}$ on $\partial \mathscr{A}$,

$$
\left[\mu\left(\operatorname{grad} \mathbf{v}_{2}\right)_{s}+\lambda\left(\operatorname{div} \mathbf{v}_{2}\right) \hat{\mathbf{I}}\right] \mathbf{N}
$$

$$
\begin{aligned}
= & \left\{\lambda\left(\mathbf{v}_{4}^{0} \cdot \mathbf{r}-w_{3}^{0}-\omega_{4}^{0} \phi\right) \hat{\mathbf{I}}\right. \\
& +\eta^{2}\left[\mu^{2}\left(\dot{\tau}^{F} \operatorname{grad} \phi+\operatorname{grad} \chi\right) \otimes \dot{\mathbf{c}}^{\prime}\right. \\
& -\mu \dot{\mathbf{c}}^{\prime} \otimes\left(\dot{\tau}^{F} * \mathbf{r}+\mathbf{K} \dot{\mathbf{c}}^{\prime}\right) \\
& -\mu \dot{\mathbf{c}}^{\prime} \vee \mathbf{v}_{0}^{\prime}-3 \lambda \theta\left(* \dot{\mathbf{c}}^{\prime} \cdot \mathbf{r}\right) \hat{\mathbf{I}} \\
& -2\left[\left(\lambda \gamma_{3}+\mu \beta_{3}\right) \delta^{2}+(\lambda+\mu) \theta^{2}\right] \hat{\mathbf{I}} \\
& +\lambda\left[\dot { \mathbf { c } } ^ { \prime } \cdot \left(\dot{\tau}^{F} \operatorname{grad} \phi+\operatorname{grad} \chi\right.\right. \\
& -2 \mathbf{v}_{0}^{\prime}-\dot{\tau}^{F}(* \mathbf{r}) \\
& \left.\left.\left.-\mathbf{K} \dot{\mathbf{c}}^{\prime}\right)\right] \hat{\mathbf{I}}\right\} \mathbf{N} \text { on } \partial \mathscr{A},
\end{aligned}
$$

where $\gamma_{3}=\left(3-6 v+6 v^{2}\right) / 2$ and $\mathscr{H}$ is the Hessian operator.

The boundary-value problem $(36)_{1}$ and $(36)_{3}$ is the standard Saint-Venant problem in linear elasticity for warping induced by flexure, and has the solution

$w_{2}=w_{2}^{0}-\mathbf{v}_{3}^{0} \cdot \mathbf{r}+\omega_{3}^{0} \phi+\mathbf{v}_{5}^{0} \cdot \mathbf{h}$.

The integrability condition (24) yields

$\mathbf{v}_{5}^{0}=\mathbf{0}$.

A general solution of $(36)_{2}$ and $(36)_{4}$ is

$\mathbf{v}_{2}=\mathbf{v}_{2}^{0}+\omega_{2}^{0}(* \mathbf{r})-v w_{3}^{0} \mathbf{r}+\mathbf{K} \mathbf{v}_{4}^{0}+\omega_{4}^{0} \tilde{\mathbf{v}}_{2}+\hat{\mathbf{v}}_{2}$,

where $\left(\omega_{4}^{0} \tilde{\mathbf{v}}_{2}+\hat{\mathbf{v}}_{2}\right)$ is a solution of $(36)_{2}$ and $(36)_{4}$ with $\mathbf{v}_{4}^{0}=\mathbf{0}$ and $w_{3}^{0}=0$. The integrability condition $(24)_{3}$ and Eq. (39) imply that

$$
\begin{aligned}
\omega_{3}^{0}= & \frac{\eta^{2}}{\left(J_{0}-D\right)}\left[2\left(\mathbf{J}\left(* \dot{\mathbf{c}}^{\prime}\right)\right) \cdot \mathbf{c}^{\prime}\right. \\
& \left.-\beta_{2}\left(* \dot{\mathbf{c}}^{\prime}\right) \cdot \int_{\mathscr{A}}(* \mathbf{r} \otimes * \mathbf{r}) \mathbf{g d} A\right] .
\end{aligned}
$$

The integrability condition $(24)_{1}$ gives

$$
\begin{aligned}
w_{3}^{0}= & -\frac{\eta^{2}}{E \mathscr{A}}\left[(\lambda+2 \mu) \frac{3 \dot{\mathbf{q}} \cdot\left(* \dot{\mathbf{c}}^{\prime}\right)}{\mu\left(J_{0}-D\right)} \int_{\mathscr{A}} \phi \mathrm{d} A\right. \\
& +3(\lambda+2 \mu)\left(\mathbf{J} \dot{\mathbf{c}}^{\prime}\right) \cdot \dot{\mathbf{c}}^{\prime} \\
& +2\left(\lambda \gamma_{1}+\mu \beta_{1}\right)\left(\mathbf{J} * \dot{\mathbf{c}}^{\prime}\right) \cdot\left(* \dot{\mathbf{c}}^{\prime}\right)
\end{aligned}
$$

$$
\begin{aligned}
& +2 \lambda\left(\dot{\tau}^{F} \mathscr{A}+v^{2}\left(\mathbf{J} \dot{\mathbf{c}}^{\prime}\right) \cdot \dot{\mathbf{c}}^{\prime}\right) \\
& +\lambda\left(\omega_{4}^{0} \int_{\mathscr{A}} \operatorname{div} \tilde{\mathbf{v}}_{2} \mathrm{~d} A\right. \\
& \left.\left.+\int_{\mathscr{A}} \operatorname{div} \hat{\mathbf{v}}_{2} \mathrm{~d} A\right)\right] .
\end{aligned}
$$

Even though the differential operators on the left-hand sides of (36) 2 and (36) 4 are those of plane linear elasticity, the source terms on the right-hand sides with $\mathbf{v}_{4}^{0}=\mathbf{0}$ and $w_{4}^{0}=0$ involve the in-plane flexural displacements and the Saint-Venant warping function $\phi$. Therefore, $\tilde{\mathbf{v}}_{2}$ and $\hat{\mathbf{v}}_{2}$ cannot, in general, be expressed in closed form. Note that $\omega_{3}^{0}$ depends upon the inertia tensor $\mathbf{J}$, the curvature of the preflexured bar, the firstorder transverse shear strains $\dot{\mathbf{g}}$ and the second-order elasticity $\beta_{2}$.

We have following Eqs. (42) for the determination of $w_{1}$ and $\mathbf{v}_{1}$ :

$$
\begin{aligned}
\mu \Delta_{R} w_{1}= & -(\lambda+\mu) \operatorname{div} \mathbf{v}_{2}-(\lambda+2 \mu) w_{3} \\
& +\eta^{2}\left\{( \lambda + \mu ) \dot { \mathbf { c } } ^ { \prime } \cdot \left[\dot{\tau}^{F}(\operatorname{grad} \phi-(* \mathbf{r}))\right.\right. \\
& \left.+\operatorname{grad} \chi-\mathbf{K} \dot{\mathbf{c}}^{\prime}-2 \mathbf{v}_{0}^{\prime}\right]-2(\lambda+\mu) \theta^{2} \\
& -\left[\lambda \gamma_{1}+\mu\left(\beta_{1}+\beta_{2}(1+v)\right)\right. \\
& +E v] \delta^{2}-\mu \dot{\mathbf{c}}^{\prime} \cdot\left(\mathbf{v}_{0}^{\prime}+\dot{\tau}^{F}(* \mathbf{r})+\mathbf{K} \dot{\mathbf{c}}^{\prime}\right) \\
& -\frac{\mu \beta_{2}}{2} \dot{\mathbf{c}}^{\prime} \cdot\left[\dot{\tau}^{F}(\operatorname{grad} \phi+* \mathbf{r})\right. \\
& \left.\left.+\operatorname{grad} \chi+\mathbf{K} \dot{\mathbf{c}}^{\prime}\right]\right\} \text { in } \mathscr{A},
\end{aligned}
$$

$$
\begin{aligned}
& \mu \Delta_{R} \mathbf{v}_{1}+(\lambda+\mu) \operatorname{grad} \operatorname{div} \mathbf{v}_{1} \\
& =-\mu \mathbf{v}_{3}-(\lambda+\mu) \operatorname{grad} w_{2} \\
& \quad-\mu \eta^{2} \theta *\left(\dot{\tau}^{F} \operatorname{grad} \phi+\nabla \chi\right. \\
& \left.\quad-\mathbf{v}_{0}^{\prime}\right) \text { in } \mathscr{A},
\end{aligned}
$$

$$
\begin{aligned}
&\left(\operatorname{grad} w_{1}\right) \cdot \mathbf{N}=\left\{-\mathbf{v}_{2}+\eta^{2}\left[\theta \left(* \mathbf{v}_{0}^{\prime}-\dot{\tau}^{F} \mathbf{r}\right.\right.\right. \\
&\left.+* \mathbf{K} \dot{\mathbf{c}}^{\prime}\right)-(1+v) \delta\left(\mathbf{v}_{0}^{\prime}+\dot{\tau}^{F}(* \mathbf{r})\right. \\
&\left.\left.\left.+\mathbf{K} \dot{\mathbf{c}}^{\prime}\right)\right]\right\} \cdot \mathbf{N} \text { on } \partial \mathscr{A} \\
& {\left[\mu\left(\operatorname{grad} \mathbf{v}_{1}\right)_{s}+\lambda\left(\operatorname{div} \mathbf{v}_{1}\right) \hat{\mathbf{I}}\right] \mathbf{N}=-\lambda w_{2} \mathbf{N} \text { on } \mathscr{A} }
\end{aligned}
$$


The structure of Eqs. (42) $)_{1}$ and (42) $)_{3}$ is the same as that of (36) 1 and (36) 3 . Proceeding in the same way as was done to obtain the solution (37) of (36) 1 and $(36)_{3}$, we can find the following solution of $(42)_{1}$ and $(42)_{3}$ :

$$
\begin{aligned}
\mathbf{v}_{1}= & \mathbf{v}_{1}^{0}+\omega_{1}^{0}(* \mathbf{r})-v w_{2}^{0} \mathbf{r}+\mathbf{K} \mathbf{v}_{3}^{0}+\omega_{3}^{0} \tilde{\mathbf{v}}_{1}+\hat{\mathbf{v}}_{1}, \\
w_{1}= & w_{1}^{0}-\mathbf{v}_{2}^{0} \cdot \mathbf{r}+\omega_{2}^{0} \phi+\mathbf{v}_{4}^{0} \cdot \mathbf{r} \\
& -w_{3}^{0} h-\omega_{4}^{0} \tilde{w}_{1}+\hat{w}_{1} .
\end{aligned}
$$

Here $\left(\omega_{3}^{0} \tilde{\mathbf{v}}_{1}+\hat{\mathbf{v}}_{1}\right)$ is a solution of $(42)_{2}$ and (42) 4 with $\mathbf{v}_{3}^{0}=\mathbf{0}$ and $\omega_{3}^{0}=0$, and $-w_{3}^{0} h-\omega_{4}^{0} \tilde{w}_{1}+\hat{w}_{1}$ is a solution of $(42)_{1}$ and (42) $)_{3}$ with $\mathbf{v}_{2}^{0}=\mathbf{0}$ and $\omega_{2}^{0}=0$. We now use compatibility conditions (24) to derive the following equations for the determination of $\mathbf{v}_{4}^{0}, w_{2}^{0}$ and $\omega_{2}^{0}$ in terms of known quantities:

$$
\begin{aligned}
2(1 & +v) * \mathbf{J} * \mathbf{v}_{4}^{0}+\int_{\mathscr{A}}\left\{\frac{\beta_{2}}{2} \delta \dot{\mathbf{g}}\right. \\
& -w_{3}^{0} \operatorname{grad} h+\omega_{4}^{0}\left(\tilde{\mathbf{v}}_{2}-\operatorname{grad} \tilde{w}_{1}\right) \\
& +\left(\hat{\mathbf{v}}_{2}+\operatorname{grad} \hat{w}_{1}\right)+[(1+v) \delta \mathbf{I} \\
& \left.+\theta *]\left(\dot{\tau}^{F} \operatorname{grad} \phi+\operatorname{grad} \chi\right)\right\}=\mathbf{0},
\end{aligned}
$$

$$
\begin{aligned}
\mu\left(J_{0}-D\right) \omega_{2}^{0}= & \dot{\mathbf{v}}_{0}^{\prime} \cdot(* \dot{\mathbf{q}}) \\
& -\mu \int_{\mathscr{A}}(* \mathbf{r}) \cdot\left[\left(\mathbf{K}+\operatorname{grad}^{\mathrm{T}} \mathbf{h}\right) \mathbf{v}_{4}^{0}\right. \\
& -w_{3}^{0} \operatorname{grad} h+\omega_{4}^{0}\left(\tilde{\mathbf{v}}_{2}-\operatorname{grad} \tilde{w}_{1}\right) \\
& \left.+\left(\hat{\mathbf{v}}_{2}+\operatorname{grad} \hat{w}_{1}\right)\right],
\end{aligned}
$$

$$
\begin{aligned}
E \mathscr{A} w_{2}^{0}= & -\omega_{3}^{0} \int_{\mathscr{A}}\left[(\lambda+2 \mu) \phi+\lambda \operatorname{div} \tilde{\mathbf{v}}_{1}\right] \\
& -\lambda \int_{\mathscr{A}}^{\operatorname{div} \tilde{\mathbf{v}}_{1} .}
\end{aligned}
$$

We have following equations for the determination of $w_{0}$ and $\mathbf{v}_{0}$ :

$\mu \Delta_{R} w_{0}=-(\lambda+\mu) \operatorname{div} \mathbf{v}_{1}-(\lambda+2 \mu) w_{2}$ in $\mathscr{A}$,

$$
\begin{aligned}
\mu \Delta_{R} \mathbf{v}_{0} & +(\lambda+\mu) \operatorname{grad} \operatorname{div} \mathbf{v}_{0} \\
= & -(\lambda+\mu) \operatorname{grad} w_{1}-\mu \mathbf{v}_{2} \\
& -\eta^{2}\left\{\mu ( 1 - v ) \delta \left(\operatorname{grad}\left(\dot{\tau}^{F} \phi+\chi\right)\right.\right. \\
& \left.-\mathbf{v}_{0}^{\prime}\right)+\frac{\mu \beta_{2}}{2} \delta \dot{\mathbf{g}}+\frac{\mu \alpha_{6}}{4} \operatorname{div}(\dot{\mathbf{g}} \otimes \dot{\mathbf{g}}) \\
& -(\lambda+\mu) \mathscr{H}(\dot{\tau} \phi+\chi)\left(\dot{\tau}^{F} * \mathbf{r}\right. \\
& \left.+\mathbf{K} \dot{\mathbf{c}}^{\prime}+\mathbf{v}_{0}^{\prime}\right)+\left(\frac{\lambda}{2}-\frac{\mu \alpha_{4}}{4}\right) \operatorname{grad}\|\dot{\mathbf{g}}\|^{2} \\
& \left.-\lambda(v \delta \hat{\mathbf{I}}-\theta *)\left(\operatorname{grad}\left(\dot{\tau}^{F} \phi+\chi\right)-\mathbf{v}_{0}^{\prime}\right)\right\} \text { in } \mathscr{A},
\end{aligned}
$$

$\left(\operatorname{grad} w_{0}\right) \cdot \mathbf{N}=-\mathbf{v}_{1} \cdot \mathbf{N}$ on $\partial \mathscr{A}$,

$$
\begin{aligned}
& {\left[\mu\left(\operatorname{grad} \mathbf{v}_{0}\right)_{s}+\lambda\left(\operatorname{div} \mathbf{v}_{0}\right) \hat{\mathbf{I}}\right] \mathbf{N} } \\
&=-\lambda w_{1} \mathbf{N}+\eta^{2}\left\{\mu\left(\operatorname{grad}\left(\dot{\tau}^{F} \phi+\chi\right)-\mathbf{v}_{0}^{\prime}\right)\right. \\
& \otimes\left(\mathbf{v}_{0}^{\prime}+\dot{\tau}^{F} * \mathbf{r}+\mathbf{K} \dot{\mathbf{c}}^{\prime}\right) \\
&+\left[( \frac { \lambda } { 2 } - \frac { \mu \alpha _ { 4 } } { 4 } ) \| \dot { \mathbf { g } } \| ^ { 2 } \left(\mathbf{v}_{0}^{\prime}+\dot{\tau}^{F} * \mathbf{r}\right.\right. \\
&\left.+\mathbf{K} \dot{\mathbf{c}}^{\prime}\right) \cdot\left(\operatorname{grad}\left(\dot{\tau}^{F} \phi+\chi\right)\right. \\
&\left.\left.\left.-\mathbf{v}_{0}^{\prime}\right)\right] \hat{\mathbf{I}}\right\} \mathbf{N} \text { on } \partial \mathscr{A} .
\end{aligned}
$$

Eqs. (48) are treated in the same way as (42) with the difference that the constants of integration $\mathbf{v}_{0}^{0}, w_{0}^{0}$ and $\omega_{0}^{0}$, and the constant $\mathbf{v}_{1}^{0}$ are evaluated from the clamping conditions (7). Thus the only unknowns left in the solution of the problem are the six constants $\mathbf{v}_{3}^{0}, \mathbf{v}_{2}^{0}, w_{1}^{0}$ and $\omega_{1}^{0}$ which characterize the second-order flexure, bending, extension and torsion respectively.

We note that all of the in-plane boundary-value problems formulated above are well-posed. Eqs. (42) 1 and $(42)_{3}$ exhibit that the source terms on their righthand sides involve $\mathbf{v}_{2}, \phi, \chi$ and $w_{3}$; a similar remark applies to $(48)_{1}$ and $(48)_{3}$. Whereas warping functions $w_{2}$ and $w_{3}$ are determined by the warping function $\phi, w_{1}$ and $w_{0}$ are solutions of new in-plane elliptic boundary-value problems. Also in-plane displacements $\mathbf{v}_{0}, \mathbf{v}_{1}$ and $\mathbf{v}_{2}$ are solutions of new inplane elliptic boundary-value problems. Expressions (40), (41), (35) and (31) for $\omega_{3}^{0}, w_{3}^{0}, \omega_{4}^{0}$ and $w_{5}^{0}$ indicate that these constants, in general, need not vanish. Thus expressions for the second-order torsion and axial elongation involve terms proportional to $z^{3}$ and $z^{4}$, and $z^{3}$ and $z^{5}$ respectively. This should not be very surprising since solution (16) for the infinitesimal flexure 
has axial elongation proportional to $z^{2}$ and in-plane displacements proportional to $z^{3}$ which make the righthand sides of equations $(23)_{1}-(23)_{4}$ polynomials in $z$ of degree four.

The non-zero constant $w_{5}^{0}$ depends on the deformations caused by the infinitesimal preflexure; thus the axial displacement of a point is proportional to $z^{5}$ where $z$ is the axial distance of the point from the end face with the clamped centroid. When the flexural vector $\dot{\mathbf{c}}^{\prime}$ is not parallel to the resultant first-order shear force $\dot{\mathbf{q}}$, the second-order twist varies as $z^{4}$ and thus can be significant for a long bar. Functions $w_{2}$ and $w_{3}$ giving the second-order axial elongations proportional to $z^{2}$ and $z^{3}$, respectively, are determined by the classical Saint-Venant warping function $\phi$. Well-posed elliptic boundary-value problems for the determination of axial and in-plane displacement functions $w_{0}, w_{1}, \ldots, w_{5}, \mathbf{v}_{0}, \mathbf{v}_{1}, \ldots, \mathbf{v}_{5}$ have been formulated and most of these functions have been explicitly determined.

For a circular cross-section, closed-form expressions for functions $\tilde{\mathbf{v}}_{2}, \hat{\mathbf{v}}_{2}, \tilde{\mathbf{v}}_{1}, \hat{\mathbf{v}}_{1}, \tilde{w}_{1}, \hat{w}_{1}, \mathbf{v}_{0}$ and $w_{0}$ can be determined and are given below.

\section{Results for a circular bar}

Let the origin of the rectangular Cartesian coordinate system be located at the center of the circular cross-section of radius $R, z$-axis along the centroidal axis of the cylinder, and $x$ - and $y$-axes along $\mathbf{c}$ and $* \mathbf{c}$ where $\mathbf{c}$ is a unit vector in a cross-section such that

$\mathbf{c}^{\prime}=\eta \mathbf{c}$.

For a circular cylinder

$\tau^{F}=0, \phi=0$

in (16). Eq. (18) can be solved for $\mathbf{h}$ and Eq. (16) simplifies to

$$
\begin{aligned}
\dot{\mathbf{u}}= & \left(\frac{\|\mathbf{r}\|^{2}}{4}-\left(\frac{3+2 v}{8} R^{2}+\frac{z^{2}}{2}\right)\right)(\mathbf{c} \cdot \mathbf{r}) \mathbf{e} \\
& +\left[z\left(\mathbf{K}-\frac{3+2 v}{8} R^{2} \hat{\mathbf{I}}\right)+\frac{z^{3}}{6} \hat{\mathbf{I}}\right] \mathbf{c} .
\end{aligned}
$$

Omitting details, the complete polynomial solution for a second-order elastic circular bar predeformed infinitesimally in flexure is given by

$$
\begin{aligned}
\ddot{w}(\mathbf{r}, z)= & -\frac{z^{5}}{40}+\frac{z^{3}}{6}\left[w_{3}^{0}+\frac{3}{2} v\left(y^{2}-x^{2}\right)\right] \\
& -\frac{z^{2}}{2}\left(\mathbf{v}_{3}^{0} \cdot \mathbf{r}\right)+z\left[w_{1}^{0}-\left(\mathbf{v}_{2}^{0} \cdot \mathbf{r}\right)+\tilde{w}_{1}(\mathbf{r})\right] \\
& +\left(\frac{2\|\mathbf{r}\|^{2}-(3+2 v) R^{2}}{8}\right)\left(\mathbf{v}_{3}^{0} \cdot \mathbf{r}\right), \\
\ddot{\mathbf{v}}(\mathbf{r}, z)= & -\frac{z^{4}}{8}(\mathbf{c} \otimes \mathbf{c}) \mathbf{r}+\frac{z^{3}}{6} \mathbf{v}_{3}^{0}+\frac{z^{2}}{2}\left[\mathbf{v}_{2}^{0}-v w_{3}^{0} \mathbf{r}+\tilde{\mathbf{v}}_{2}(\mathbf{r})\right] \\
& +z\left[-\frac{a}{2} \mathbf{v}_{3}^{0}+\omega_{1}(* \mathbf{r})+\mathbf{K} \mathbf{v}_{3}^{0}\right] \\
& -v w_{1}^{0} \mathbf{r}+\mathbf{K} \mathbf{v}_{2}^{0}+\tilde{\mathbf{v}}_{0}(\mathbf{r}) .
\end{aligned}
$$

It depends on the undetermined kinematic constants $w_{1}^{0}, \mathbf{v}_{2}^{0}, \mathbf{v}_{3}^{0}, \omega_{1}$ defining second-order extension, bending, flexure and torsion superimposed on the firstorder extension, bending, flexure and torsion respectively. Functions $\tilde{\mathbf{v}}_{2}(r), \tilde{\omega}_{1}(r)$ and $\tilde{\mathbf{v}}_{0}(r)$ are parts of $\mathbf{v}_{2}(r), \omega_{1}(r)$ and $\mathbf{v}_{0}(r)$ that do not represent a rigid body motion and do not depend upon constants $\omega_{3}^{0}$, $\mathbf{v}_{4}^{0}$ and $\mathbf{v}_{2}^{0}$. With the rectangular Cartesian coordinates $(x, y, z)$ aligned along $(\mathbf{c}, * \mathbf{c}, \mathbf{e})$, these functions exhibit following symmetries.

$$
\begin{aligned}
& \tilde{v}_{21}(x, y)=-\tilde{v}_{21}(-x, y), \quad \tilde{v}_{22}(x, y)=-\tilde{v}_{22}(-x, y), \\
& \tilde{v}_{21}(x, y)=\tilde{v}_{21}(x,-y), \quad \tilde{v}_{22}(x, y)=-\tilde{v}_{22}(x,-y), \\
& \tilde{w}_{1}(x, y)=\tilde{w}_{1}(-x, y), \quad \tilde{w}_{1}(x, y)=w_{1}(x,-y), \\
& \tilde{v}_{01}(x, y)=-\tilde{v}_{01}(-x, y), \quad \tilde{v}_{02}(x, y)=\tilde{v}_{02}(-x, y), \\
& \tilde{v}_{01}(x, y)=\tilde{v}_{01}(x,-y), \quad \tilde{v}_{02}(x, y)=-\tilde{v}_{02}(x,-y) .
\end{aligned}
$$

With the notations

$$
\begin{aligned}
& P(\mathbf{v})=\mu \Delta \mathbf{v}+(\lambda+\mu) \operatorname{grad}(\operatorname{div} \mathbf{v}) \text { in } \mathscr{A}, \\
& B(\mathbf{v})=\left[\mu(\operatorname{grad} \mathbf{v})_{s}+(\lambda \operatorname{div} \mathbf{v}) \hat{\mathbf{I}}\right] \mathbf{N} \text { on } \partial \mathscr{A}, \\
& \dot{\mathbf{g}}=\dot{\mathbf{v}}^{\prime}+\operatorname{grad} \dot{w}
\end{aligned}
$$

field equations for the determination of $\tilde{\mathbf{v}}_{2}, \tilde{w}_{1}$ and $\tilde{\mathbf{v}}_{0}$ are:

$$
\begin{aligned}
P\left(\tilde{\mathbf{v}}_{2}\right)= & \{[5(\lambda+\mu)+2 \mu(2-v)] \mathbf{c} \otimes \mathbf{c} \\
& +[\mu-2 \lambda(1+v)] * \mathbf{c} \otimes * \mathbf{c}\} \frac{\mathbf{r}}{2} \quad \text { in } \mathscr{A}, \\
B\left(\tilde{\mathbf{v}}_{2}\right)= & \mu[\mathbf{c} \otimes(\mathbf{A c})-(\mathbf{K c}) \otimes \mathbf{c}] \mathbf{n} \\
& +\left\{\lambda\left[\frac{3+4 v}{4}-2\left(\lambda \gamma_{3}+\mu \beta_{3}\right)\right] \delta^{2}\right. \\
& \left.+\frac{1-8 v}{4 v^{2}} \lambda \theta^{2}\right\} \times \mathbf{N} \quad \text { in } \partial \mathscr{A},
\end{aligned}
$$


$P\left(\tilde{w}_{1}\right)=0$ in $\mathscr{A}$,

$B\left(\tilde{w}_{1}\right)=0$ on $\partial \mathscr{A}$,

$P\left(\tilde{\mathbf{v}}_{0}\right)=$

$-\mu \mathbf{v}_{2}(\mathbf{r})-(\lambda+\mu) \operatorname{grad} w_{1}(\mathbf{r})-\mu \frac{\beta_{2}}{2} \delta \dot{\mathbf{g}}$

$-\mu \frac{\alpha_{6}}{4} \operatorname{div}(\dot{\mathbf{g}} \otimes \dot{\mathbf{g}})+\beta_{4} \operatorname{div}\left(\|\dot{\mathbf{g}}\|^{2} \hat{\mathbf{I}}\right)$

$+\mu \operatorname{div}\left\{(\mathbf{K c}) \otimes(\mathbf{A c})-\frac{a}{2}[\mathbf{c} \otimes(\mathbf{A c})+(\mathbf{K c}) \otimes \mathbf{c}]\right\}$

$-\frac{\lambda}{8} \operatorname{grad}\left\{a\left[(3+2 v) \delta^{2}+\frac{1-2 v}{v^{2}} \theta^{2}\right]\right.$

$\left.-v\|\mathbf{r}\|^{2}\left(3 \delta^{2}-\frac{\theta^{2}}{v^{2}}\right)\right\} \quad$ in $\mathscr{A}$,

$B\left(\tilde{\mathbf{v}}_{0}\right)=\left\{\mu \frac{a^{2}}{4}(\mathbf{c} \otimes \mathbf{c})-\lambda\left[w_{1}(\mathbf{r})+\frac{a^{2}}{4}+\beta_{4}\|\dot{\mathbf{g}}\|^{2}\right] \hat{\mathbf{I}}\right\} \mathbf{N}$ $+\frac{\mu}{2}\{(\mathbf{K c}) \otimes(\mathbf{A c})-a[\mathbf{c} \otimes(\mathbf{A c})+(\mathbf{K c}) \otimes \mathbf{c}]\} \mathbf{N}$

$-\frac{\lambda}{8}\left\{a\left[(3+2 v) \delta^{2}+\frac{1-2 v}{v^{2}} \theta^{2}\right]\right.$ $\left.-v\|\mathbf{r}\|^{2}\left(3 \delta^{2}-\frac{\theta^{2}}{v^{2}}\right)\right\} \mathbf{N}$ in $\partial \mathscr{A}$,

where

$\mathbf{A}=\frac{3 \mathbf{r} \otimes \mathbf{r}+* \mathbf{r} \otimes * \mathbf{r}}{4}, \theta=v(* \mathbf{c}) \cdot \mathbf{r}$.

Note that $\dot{\mathbf{g}}$ defined by $(54)_{3}$ equals the first-order shear strain; henceforth this definition of $\dot{\mathbf{g}}$ rather than that given by $(19)_{2}$ is used. Each of these boundary value problems defined by Eqs. (55)-(58) are well posed. Their solutions are

$$
\begin{aligned}
\tilde{v}_{21}= & b_{1} x+b_{2} x y^{2}+b_{3} x^{3}, \\
\tilde{v}_{22}= & b_{4} y+b_{5} x^{2} y+b_{6} y^{3}, \\
D \tilde{w}_{1} & (x, y) \\
= & x^{2} R^{2}\left[144 \lambda^{6}+942 \lambda^{5} \mu+2726 \lambda^{4} \mu^{2}\right. \\
& +4486 \lambda^{3} \mu^{3}+4124 \lambda^{2} \mu^{4}+1814 \lambda \mu^{5}+260 \mu^{6} \\
& +16 \mu(\lambda+\mu)^{3}(\lambda+2 \mu)(9 \lambda+7 \mu) \beta_{1} \\
& +2 \mu(\lambda+\mu)^{2}(28 \lambda+19 \mu)\left(3 \lambda^{2}+8 \lambda \mu\right.
\end{aligned}
$$

$$
\begin{aligned}
& \left.+4 \mu^{2}\right) \beta_{2}+\left(48 \lambda^{5} \mu+384 \lambda^{4} \mu^{2}+1136 \lambda^{3} \mu^{3}\right. \\
& \left.\left.+1568 \lambda^{2} \mu^{4}+1024 \lambda \mu^{5}+256 \mu^{6}\right) \beta_{3}\right] \\
& +y^{2} R^{2}\left[30 \lambda^{5} \mu+82 \lambda^{4} \mu^{2}+74 \lambda^{3} \mu^{3}\right. \\
& +76 \lambda^{2} \mu^{4}+70 \lambda \mu^{5}+4 \mu^{6} \\
& -16 \mu(\lambda+\mu)^{3}(3 \lambda+\mu)(\lambda+2 \mu) \beta_{1} \\
& -2 \mu(\lambda+\mu)^{2}(4 \lambda+\mu)\left(3 \lambda^{2}+8 \lambda \mu+4 \mu^{2}\right) \beta_{2} \\
& +\left(48 \lambda^{5} \mu+288 \lambda^{4} \mu^{2}+688 \lambda^{3} \mu^{3}+832 \lambda^{2} \mu^{4}\right. \\
& \left.\left.+512 \lambda \mu^{5}+128 \mu^{6}\right) \beta_{3}\right] \\
& +x^{2} y^{2}\left[-54 \lambda^{6}-270 \lambda^{5} \mu-552 \lambda^{4} \mu^{2}\right. \\
& -660 \lambda^{3} \mu^{3}-462 \lambda^{2} \mu^{4}-150 \lambda \mu^{5}-12 \mu^{6} \\
& -24 \mu(\lambda+\mu)^{3}(\lambda+2 \mu)(3 \lambda+2 \mu) \beta_{1} \\
& +\left[\mu(\lambda+\mu)^{2}(4 \lambda+\mu)\right. \\
& \left.-\mu(\lambda+\mu)^{2}(28 \lambda+19 \mu)\right]\left(3 \lambda^{2}+8 \lambda \mu\right. \\
& \left.+4 \mu^{2}\right) \beta_{2}+x^{4}\left[-27 \lambda^{6}-51 \lambda^{5} \mu+104 \lambda^{4} \mu^{2}\right. \\
& +270 \lambda^{3} \mu^{3}+169 \lambda^{2} \mu^{4}+21 \lambda \mu^{5}-6 \mu^{6} \\
& -28 \mu(\lambda+\mu)^{3}(\lambda+2 \mu)(3 \lambda+2 \mu) \beta_{1} \\
& -\mu(\lambda+\mu)^{2}(28 \lambda+19 \mu)\left(3 \lambda^{2}+8 \lambda \mu\right. \\
& \left.+4 \mu^{2}\right) \beta_{2}+\left(48 \lambda^{5} \mu+272 \lambda^{4} \mu^{2}+592 \lambda^{3} \mu^{3}\right. \\
& \left.\left.+624 \lambda^{2} \mu^{4}+320 \lambda \mu^{5}+64 \mu^{6}\right) \beta_{3}\right] \\
& +y^{4}\left[-27 \lambda^{6}-219 \lambda^{5} \mu-656 \lambda^{4} \mu^{2}-930 \lambda^{3} \mu^{3}\right. \\
& -631 \lambda^{2} \mu^{4}-171 \lambda \mu^{5}-6 \mu^{6} \\
& +4 \mu(\lambda+\mu)^{3}(\lambda+2 \mu)(3 \lambda+2 \mu) \beta_{1} \\
& +\mu(\lambda+\mu)^{2}(4 \lambda+\mu)\left(3 \lambda^{2}+8 \lambda \mu+4 \mu^{2}\right) \beta_{2} \\
& -\left(48 \lambda^{5} \mu+272 \lambda^{4} \mu^{2}+592 \lambda^{3} \mu^{3}+624 \lambda^{2} \mu^{4}\right. \\
& \left.\left.+320 \lambda \mu^{5}+64 \mu^{6}\right) \beta_{3}\right] . \\
&
\end{aligned}
$$

$$
\begin{aligned}
\tilde{v}_{01}(x, y)= & d_{1} x+d_{2} x y^{2}+d_{3} x^{3} \\
& +d_{4} x y^{4}+d_{5} x^{3} y^{2}+d_{6} x^{5} \\
\tilde{v}_{22}(x, y)= & d_{7} y+d_{8} x^{2} y+d_{9} y^{3} \\
& +d_{10} x^{4} y+d_{11} x^{2} y^{3}+d_{12} y^{5}
\end{aligned}
$$

where

$$
\begin{aligned}
D= & 192 \mu(\lambda+\mu)^{3}(\lambda+2 \mu)(3 \lambda+2 \mu), \\
b_{1}= & -\frac{R^{2}}{32 \mu(\lambda+\mu)(\lambda+2 \mu)}\left[2 \mu^{3}(19+v)\right. \\
& +2 \lambda^{3}\left(17-34 v+24 v^{2}\right) \\
& +3 \lambda \mu^{2}\left(51-74 v+64 v^{2}\right) \\
& +2 \lambda^{2} \mu\left(67-124 v+96 v^{2}\right) \\
& \left.+16 \mu(\lambda+2 \mu)^{2} \beta_{3}\right],
\end{aligned}
$$




$$
\begin{aligned}
b_{2}= & \frac{1}{32 \mu(\lambda+\mu)(\lambda+2 \mu)}\left[2 \mu^{3}(13-5 v)\right. \\
& +2 \lambda^{3}\left(17-34 v+24 v^{2}\right) \\
& +\lambda \mu^{2}\left(153-296 v+192 v^{2}\right) \\
& +\lambda^{2} \mu\left(137-282 v+192 v^{2}\right) \\
& \left.+16 \mu(\lambda+2 \mu)^{2} \beta_{3}\right], \\
b_{3}= & \frac{1}{96 \mu(\lambda+\mu)(\lambda+2 \mu)}\left[2 \mu^{3}(31-7 v)\right. \\
& +2 \lambda^{3}\left(17-34 v+24 v^{2}\right) \\
& +\lambda \mu^{2}(107-16 v)+\lambda^{2} \mu\left(103-142 v+96 v^{2}\right) \\
& \left.+16 \lambda \mu(\lambda+2 \mu) \beta_{3}\right],
\end{aligned}
$$$$
b_{4}=\frac{R^{2}}{32 \mu(\lambda+\mu)(\lambda+2 \mu)}\left[2 \mu^{3}(1-v)\right.
$$$$
+2 \lambda^{3}\left(17-34 v+24 v^{2}\right)
$$$$
+\lambda \mu^{2}(43-50 v)+2 \lambda^{2} \mu\left(45-80 v+48 v^{2}\right)
$$$$
\left.+16 \lambda \mu(\lambda+2 \mu) \beta_{3}\right] \text {, }
$$

$$
\begin{aligned}
b_{5}= & \frac{1}{32 \mu(\lambda+\mu)(\lambda+2 \mu)}\left[6 \mu^{3}(-1+v)\right. \\
& -2 \lambda^{3}\left(17-34 v+24 v^{2}\right) \\
& +\lambda^{2} \mu\left(-131+278 v-192 v^{2}\right) \\
& +\lambda \mu^{2}\left(-127+288 v-192 v^{2}\right) \\
& \left.-16 \mu(\lambda+2 \mu)^{2} \beta_{3}\right],
\end{aligned}
$$

$$
\begin{aligned}
b_{6}= & \frac{1}{96 \mu(\lambda+\mu)(\lambda+2 \mu)}\left[2 \mu^{3}(-1+v)\right. \\
& -2 \lambda^{3}\left(17-34 v+24 v^{2}\right) \\
& -\lambda \mu^{2}(29+8 v)+\lambda^{2} \mu\left(-85+130 v-96 v^{2}\right) \\
& \left.-16 \lambda \mu(\lambda+2 \mu) \beta_{3}\right] .
\end{aligned}
$$

Lengthy expressions for constants $d_{1}, d_{2}, \ldots, d_{12}$ obtained with Mathematica are omitted for the sake of brevity. Functions $\tilde{\mathbf{v}}_{0}, \tilde{w}_{1}$ and $\tilde{\mathbf{v}}_{2}$ represent generalized Poynting effects.

The following expressions for the second-order resultant forces and couples on the cross-section $\mathscr{A}$ reveal that the resultant second-order shear force and the resultant second-order moments are linear in $\mathbf{v}_{2}^{0}, \mathbf{v}_{3}^{0}$ and $\omega_{1}$, while the resultant second-order axial force is an affine function of $w_{1}^{0}$.

$$
\begin{aligned}
& \int_{\mathscr{A}} \ddot{\sigma}= \mu(1+v) A w_{1}^{0} \\
&+\frac{\pi R^{6}}{384(\lambda+\mu)^{3}(\lambda+2 \mu)}\left[52 \lambda^{5}+372 \lambda^{4} \mu\right. \\
&+978 \lambda^{3} \mu^{2}+1279 \lambda^{2} \mu^{3}+874 \lambda \mu^{4}+240 \mu^{5} \\
&+16 \mu^{3}\left(5 \lambda^{2}+18 \lambda \mu+16 \mu^{2}\right) \alpha_{3} \\
&-4 \lambda \mu\left(7 \lambda^{3}+55 \lambda^{2} \mu+115 \lambda \mu^{2}+66 \mu^{3}\right) \alpha_{4} \\
&+\left(32 \lambda^{3} \mu^{2}+114 \lambda^{2} \mu^{3}+144 \lambda \mu^{4}+88 \mu^{5}\right) \alpha_{5} \\
&+\left(24 \lambda^{4} \mu+167 \lambda^{3} \mu^{2}+360 \lambda^{2} \mu^{3} 288 \lambda \mu^{4}\right. \\
&\left.\left.+88 \mu^{5}\right) \alpha_{6}\right], \\
& \int_{\mathscr{A}} \ddot{\mathbf{t}}= 2 \mu(1+v)(* \mathbf{J} *) \mathbf{v}_{3}^{0}, \\
& \int_{\mathscr{A}}(* \mathbf{r}) \cdot \ddot{\mathbf{t}}=\mu J_{0} \omega_{1}, \\
& \int_{\mathscr{A}}(-\ddot{\sigma} * \mathbf{r})=2 \mu(1+v) \mathbf{J}\left(* \mathbf{v}_{2}^{0}\right) .
\end{aligned}
$$

We note the resultant second-order shear force and the total torque do not depend on second-order elasticities but only on the pertinent kinematical parameters. However, the second-order axial force is strongly influenced by all of the second-order elasticities. Eq. (64) 1 implies that even when the second-order and hence the total axial force vanishes, the second-order axial deformation $w_{1}^{0}$ is not zero; its value is determined by the first- and second-order elasticities. It generalizes Rivlin's result for the axial deformation in a pretwisted bar to that in a preflexured bar.

The complete solution of the problem is obtained by substituting from (51) and (52) into (10) 1 . It can be used to design experiments for determining secondorder elasticities for homogeneous and isotropic materials. Indeed, consider extension superimposed on flexure, i.e., let $\mathbf{v}_{2}^{0}, \mathbf{v}_{3}^{0}, \omega_{1}=0$. Then by measuring the resultant second-order traction and the elongation at different sections, it should be possible to evaluate the non-dimensional elasticities $\alpha_{3}, \alpha_{4}, \alpha_{5}, \alpha_{6}$ from Eqs. (52) and (64).

\section{Conclusions}

We have analyzed Saint-Venant's problem for an isotropic and homogeneous second-order elastic pris- 
matic bar predeformed in flexure. For a general crosssection, the solution has been expressed in terms of well-posed boundary value problems. For a circular bar, these boundary value problems have been solved and a complete solution in terms of six constants characterizing second-order extension, bending, flexure and torsion superimposed on first-order flexure is given. The second-order displacement is a polynomial in both the axial and the inplane coordinates. It depends on the first- and the second-order elasticities. Expressions for the resultant tractions and moments provide means for experimentally evaluating the second-order elasticities. Only the second-order axial force depends upon second-order elasticities; this relation can be used to experimentally find second-order elasticities. Eq. (64) 1 generalizes Rivlin's result for the axial deformation in a pretwisted bar to that in a preflexured bar; it thus represents a generalized Poynting effect. It is clear from Eqs. (51) and (52) that the first-order and the second-order inplane displacements involve terms multiplying $z^{3}$ and $z^{4}$ respectively; the corresponding axial displacement has terms multiplying $z^{2}$ and $z^{5}$. Thus the first-order and the second-order Poisson effects are quite different.

\section{Acknowledgements}

Giuseppe C. Ruta gratefully acknowledges the support of the "Progetto giovani ricercatori anno 2002" of the University of Roma "La Sapienza".

\section{References}

[1] J.H. Poynting, On pressure perpendicular to the shear-planes in finite pure shears, and on the lengthening of loaded wires when twisted, Proc. Roy. Soc. London A82 (1909) 546-549.

[2] R.S. Rivlin, The solution of problems in second order elasticity theory, J. Rational Mechs. Analysis 2 (1953) 53-81.

[3] C.A. Truesdell, W. Noll, The Nonlinear Field Theories of Mechanics, in: S. Flügge (Ed.), Handbuch der Physik, Vol. III/3, Springer, Berlin, 1965.
[4] C.-C. Wang, C.A. Truesdell, Introduction to Rational Elasticity, Noordhoff Int. Publishing, Leyden, 1973.

[5] A.-J.-C.B. Saint-Venant, Memóire sur la torsion des prismes, Mé́oires des Savants étrangers 14 (1856) 233.

[6] A.-J.-C.B. Saint-Venant, Memóire sur la flexion des prismes, J. de Mathématiques de Liouville, Ser. II 1 (1856) 89.

[7] D. Iesan, Saint-Venant's problem for inhomogeneous and anisotropic elastic bodies, J. Elasticity 6 (1976) 277-294.

[8] D. Iesan, On Saint-Venant's problem for elastic dielectrics, J. Elasticity 21 (1989) 101.

[9] D. Iesan, Saint-Venant Problem, Springer, New York NY, 1987.

[10] D. Iesan, L. Nappa, Saint-Venant's problem for microstretch elastic solids, Int. J. Engng. Sci. 32 (1994) 229-236.

[11] F. dell'Isola, L. Rosa, Saint-Venant Problem in Linear Piezoelectricity, in: V.V. Varadhan (Ed.), Mathematics and Control in Smart Structures, SPIE, Vol. 2715, February 1996, pp. 399-409.

[12] F. Daví, Saint Venant's problem for linear piezoelectric bodies, J. Elasticity 43 (1996) 227-245.

[13] F. dell'Isola, G.C. Ruta, R.C. Batra, Generalized Poynting effects in predeformed-prismatic bars, J. Elasticity 50 (1998) 181-196.

[14] F. dell'Isola, G.C. Ruta, R.C. Batra, A second-order solution of Saint-Venant's problem for an elastic pretwisted bar using Signorini's perturbation method, J. Elasticity 49 (1998) $113-127$.

[15] A. Signorini, Sulle deformazioni termoelastiche finite, Proc. 3rd Int. Congr. Appl. Mechs. 2 (1930) 80-89.

[16] A.E. Green, J.E. Adkins, Large Elastic Deformations and Nonlinear Continuum Mechanics, Claredon Press, Oxford, 1960.

[17] R.C. Batra, F. dell'Isola, S. Vidoli, A second-order solution of Saint-Venant's problem for a piezoelectric circular bar using Signorini's perturbation method, J. Elasticity 52 (1998) 75-90.

[18] S. Vidoli, R.C. Batra, F. dell'Isola, Saint-Venant problem for a second-order piezoelectric prismatic bar, Int. J. Engng. Sci. 38 (1999) 21-45.

[19] G. Capriz, P. Podio Guidugli, The role of Fredholm conditions in Signorini's perturbation method, Archive for Rational Mechanics and Analysis 70 (1979) 261-288.

[20] E. Trefftz, Über den Schubmittelpunkt in einem durch eine Einzellast gebogenen Balken, ZAMM 15 (1935) 220-226.

[21] G.C. Ruta, Il Problema di Saint-Venant, Studi e Ricerche del Dipartimento di Ingegneria Strutturale e Geotecnica dell’Università “La Sapienza” di Roma 7 (1995). 